\title{
L'immersion fictionnelle, ou comment concilier art et émotion en cours de langue
}

Catherine Muller, Université Grenoble Alpes, LIDILEM, France

\section{Introduction}

Selon le Livre blanc sur le dialogue interculturel, les arts « traversent naturellement les frontières, établissent des connexions et parlent directement aux émotions des personnes » (36). L'introduction de supports artistiques en classe de langue permet ainsi d'éveiller l'imaginaire des apprenants et de susciter chez eux des émotions qui se traduiront par des formes d'expression personnelle.

Notre contribution s'appuie sur un dispositif que nous avons mis en place en classe de français enseigné comme langue étrangère et qui a consisté à montrer des photographies d'auteur pour déclencher des interactions verbales entre les apprenants (Muller, « Paroles »). La perspective adoptée s'attache à la réception de ces images par les étudiants, c'est-à-dire aux différentes réactions verbales, comme la narration, la description, l'interprétation ou encore les jugements de gouts.

Nous nous concentrerons plus particulièrement sur l'une des réactions des apprenants, celle de l'immersion fictionnelle, à travers laquelle les étudiants s'approprient la photographie. De la même manière qu'un lecteur entre dans l'univers d'un roman ou un spectateur dans l'univers d'un film, les apprenants peuvent entrer eux aussi dans l'univers des photographies en réagissant émotionnellement et affectivement aux scènes représentées sur les images. Ce phénomène témoigne de leur appropriation de la photographie : les étudiants font comme si les personnages étaient eux-mêmes, comme si ces photographies étaient les leurs. C'est ainsi qu'ils sont amenés à prêter leur voix aux personnages (Muller, « Déclencheur »). Ils deviennent alors acteurs des images.

Après avoir présenté le dispositif didactique mis en place, nous nous attacherons à la notion d'immersion fictionnelle. L'approche énonciative sélectionnée pour l'étude du corpus fera l'objet de la troisième section, avant l'analyse de l'attribution par les apprenants de paroles aux personnages des photographies. 


\section{Présentation du dispositif didactique : la photographie d'auteur comme déclencheur d'interactions}

Dans le cadre d'une participation observante, nous avons montré douze photographies d'art et de presse à un total de 98 apprenants adultes de 43 nationalités différentes. Nous nous situons ici dans une approche artistique en contexte pluriculturel (Muller, "Rencontre »). Le dispositif a été mené en contexte associatif et universitaire dans des cours de français enseigné comme langue étrangère à Paris. Au total, huit groupes de niveaux A1 à B2 y ont participé. Les échanges verbaux produits dans le cadre de l'activité ont été enregistrés puis transcrits, ce qui a permis la constitution d'un corpus de 51 séquences de commentaires de photographies.

Le projet repose sur le fondement suivant : la photographie d'auteur, en tant qu'objet culturel fictionnel, peut apporter des « enjeux communicatifs » (Moore et Simon) en classe de langue (Muller, «Outil»). Les images ont été utilisées comme des déclencheurs d'interactions. Aucune information n'a été apportée sur leur signification, leur localisation, leur titre ou leur auteur. L'activité proposée constitue une technique didactique qui peut intervenir de façon ponctuelle dans les cours de langue (Beacco 17). Elle ne s'inscrit pas dans une méthodologie constituée. Bien qu'aucun objectif langagier n'ait volontairement été prédéterminé, cette activité a facilité l'appropriation par les apprenants des structures des interactions sociales ordinaires.

Nous nous focaliserons pour l'étude de l'immersion fictionnelle sur les réactions des apprenants à l'observation de la photographie Les mariés ${ }^{1}$, réalisée en 1971 par Arthur Tress, un artiste américain qui propose une vision personnelle empreinte d'imaginaire. On se situe du côté de la mise en scène avec un décor théâtral où les objets du quotidien sont subvertis, ce qui a pour effets de perturber et d'intriguer le spectateur. Cette photographie a suscité chez les apprenants des réactions d'immersion fictionnelle.

\section{L'immersion fictionnelle comme forme de réception artistique}

Le phénomène d'immersion fictionnelle (Schaeffer ; Esquenazi) consiste à entrer dans un univers de fiction, qu'il s'agisse d'un roman, d'un film ou d'une pièce de théâtre, jusqu'à oublier temporairement le monde réel. Avant d'aborder cette entrée dans l'univers fictionnel, il sera nécessaire de présenter la notion de fiction. 


\section{1- La notion de fiction}

Comme l'a mis en évidence John Searle, la fiction repose sur une suspension des conventions d'assertion habituelles. Les romans ne sont pas tenus de dire la vérité. Les écrivains racontent des histoires fictionnelles sans qu'on les traite de menteurs. On a affaire à une feinte partagée : l'auteur de fiction feint d'asserter sans pour autant vouloir tromper son destinataire. La feintise ludique consiste à faire «comme si ». La fiction présente des similarités avec le monde réel mais ce mimétisme n'a pas pour visée d'induire le destinataire à confondre univers imaginaire et univers réel (Schaeffer 156).

La feintise ludique n'exclut donc pas une conscience de la fiction chez le récepteur. C'est ce que souligne Bernard Lahire : « Le lecteur peut ainsi 'faire comme si' il lisait des histoires réelles, vraies, authentiques tout en n'étant jamais complètement dupe de leur caractère fictif » (110). Jean-Jacques Wunenburger met également en évidence le rôle de la conscience qui permet de ne pas confondre représentation et représenté (85).

\section{2-L'entrée dans l'univers fictionnel}

L'entrée dans l'univers fictionnel est facilitée par l'analogie perçue entre l'univers fictionnel et le monde réel des récepteurs. Cette similitude n'a pas besoin d'être exacte pour que les destinataires participent de façon imaginaire à la fiction (Lahire 112). Si l'analogie entre le monde réel et l'univers fictionnel touche aux intérêts du destinataire, ce dernier pourra alors considérer la fiction comme «une paraphrase de sa propre existence» (Esquenazi 18) et s'approprier l'univers fictionnel grâce à la médiation des personnages. Lorsque l'immersion fictionnelle se produit, on peut dire que le récepteur est captivé par l'univers fictionnel, ce qui lui permet d'oublier temporairement sa vie actuelle (10). Il se laisse prendre par la fiction, bien qu'il ait su, avant de s'y exposer, qu'il ne s'agissait que de simulacres (Schaeffer 22-23).

Le phénomène d'immersion fictionnelle, fréquemment étudié pour la lecture de romans ou le visionnage de films, peut être appliqué à l'observation de photographies. On peut en effet considérer la photographie comme une forme de fiction dont le caractère analogique facilite l'entrée dans l'univers. Pour que l'immersion fictionnelle opère, le jeu consiste à faire «comme si l'image était réelle » (Wunenburger 85). Cependant, l'image visuelle fixe nécessite « une intervention active de mon imaginaire » (Schaeffer 249).

C'est ainsi que les apprenants sont amenés à entrer dans l'univers fictionnel des photographies présentées lorsqu'ils perçoivent une analogie entre leur existence et certains traits de l'univers photographique. Leurs réactions témoignent d'une empathie envers les 
personnages représentés, qui se manifeste par la façon dont ils leur prêtent leur voix.

\section{2- Méthode d'analyse : une étude énonciative du corpus}

Nous nous appuierons pour l'analyse du corpus sur les spécificités de l'énonciation en classe de langue, ainsi que sur la notion de polyphonie énonciative.

\section{1- L'énonciation en classe de langue}

La communication en classe de langue et la fiction littéraire présentent des similarités. En effet, toutes deux transgressent la règle tacite de l'échange qui demande aux locuteurs de rendre leur contribution véridique (Grice 61). Anne Trévise a mis en évidence la présence dans la classe de langue d'une énonciation «simulée » ou «translatée » lors de la réalisation d'exercices ou de jeux de rôles. On observe une virtualité des locuteurs et une "pseudoréférence » (Cicurel 81) au niveau des pronoms personnels.

Ce phénomène de fiction apparait lorsque enseignant et apprenants assument une identité fictive et construisent des univers imaginaires. Les participants ont recours à la feintise ludique : ils jouent à être quelqu'un d'autre, ils font « comme si » ils se trouvaient en un autre lieu que dans la classe. Cette communication fictionnelle favorise la création d'univers se détachant de l'environnement de classe.

Les photographies déclenchent des créations fictionnelles de façon spécifique en encourageant les apprenants à faire comme s'ils étaient les sujets photographiés et pouvaient leur prêter leur voix. Ils ne se réfèrent pas à une situation entièrement imaginaire, mais à la scène représentée sur l'image.

\section{2- La polyphonie énonciative}

La communication fictionnelle de la classe de langue peut être analysée à l'aide de la notion de polyphonie énonciative. Celle-ci a été étudiée par Oswald Ducrot dans les échanges quotidiens : derrière un «je» peut se cacher un autre «je». L'auteur distingue trois instances : le sujet parlant, le locuteur et l'énonciateur :

\begin{tabular}{|c|c|c|}
\hline Émetteur & Récepteur & Dans le corpus \\
\hline $\begin{array}{c}\text { Sujet parlant } \\
\text { être empirique » }\end{array}$ & Auditeur & Apprenant en classe \\
\hline $\begin{array}{c}\text { Locuteur } \\
\text { responsable de l'énoncé, metteur en } \\
\text { scène }\end{array}$ & Allocutaire & $\begin{array}{c}\text { Spectateur commentant une } \\
\text { photographie }\end{array}$ \\
\hline $\begin{array}{c}\text { Énonciateur } \\
\text { présente son point de vue }\end{array}$ & Destinataire & Personnage des photographies \\
\hline
\end{tabular}


Le sujet parlant correspond à l'individu qui produit l'énoncé. L'auditeur lui fait face. Dans le corpus, on peut considérer que l'apprenant constitue le sujet parlant; il s'agit de l' «être empirique » (Ducrot 199) qui se situe dans la classe de français.

En tant que responsable de l'énoncé, le locuteur peut mettre en scène des énonciateurs. Le narrateur constitue son équivalent littéraire (207). L'allocutaire en constitue le pendant. Dans le corpus, le locuteur peut être assimilé au spectateur qui commente la photographie.

Les énonciateurs mis en scène par le locuteur présentent leur point de vue. Ils font face aux destinataires. Dans le corpus, les énonciateurs sont les personnages sur les photographies auxquels les apprenants prêtent leur voix.

Pour étudier la polyphonie dans le corpus, il sera nécessaire d'analyser les différentes sources énonciatives dans le discours des apprenants lorsqu'ils attribuent des paroles aux sujets photographiés.

\section{3- Analyse des données : l'attribution de paroles aux personnages des photographies}

L'immersion fictionnelle des apprenants se traduit par l'attribution de paroles aux personnages des images. Elle se manifeste par l'emploi du discours direct rapporté, à travers lequel les étudiants deviennent metteurs en scène des photographies. Lorsque les paroles ne sont précédées d'aucun verbe introducteur, ils apparaissent comme des acteurs des photographies $^{2}$.

\section{1- Discours direct rapporté : les apprenants, metteurs en scène des photographies}

Ducrot met en exergue la présence de plusieurs responsables, c'est-à-dire de plusieurs locuteurs, dans le discours direct rapporté, qui constitue un exemple de double énonciation (193). Bien que le discours direct rapporté représente une reprise de paroles, une correspondance littérale des paroles n'apparait pas nécessairement. Catherine Boré souligne la difficulté de déterminer avec exactitude les paroles rapportées ; souvent, on peut douter de « l'existence antérieure de paroles prétendument rapportées » (93). Dans le corpus étudié, les apprenants ne rapportent pas des paroles qui auraient été émises précédemment. Leurs énoncés relèvent de leur seule imagination (Muller). Les étudiants jouent ainsi le rôle de metteur en scène ou de dramaturge : ce sont eux qui attribuent des paroles aux personnages, en les introduisant par un verbe support. 
C'est le cas de Nasiba dont le tour de parole comporte plusieurs segments :

Exemple 1

SEQ 27 :

122 Nasiba euh il fait la main comme ça (mime le geste de la main sur la photo) il dit euh je veux vivre avec toi toute la vie (petit rire) euh toute mon vie euh oui peut-être parce que il +

L'apprenante décrit tout d'abord la position de la main du sujet photographié : «il fait la main comme ça ». C'est ce geste qui l'encourage à attribuer des paroles au personnage. Le passage du « $i l »$ au «je » est marqué par le verbe introducteur « dire » et par une hésitation «euh» : « il dit euh ». Les paroles rapportées apparaissent dans le segment suivant : « je veux vivre avec toi toute la vie ». Le petit rire de Nasiba marque le passage de l'énonciateur personnage de la photographie à l'énonciatrice apprenante en cours de langue. On observe une focalisation sur la langue et non plus sur le contenu dans l'extrait " euh toute mon vie »: le locuteur n'est plus le spectateur de la photographie mais l'étudiante en classe. Cependant, ce segment «toute mon vie » est attribué au personnage de la photographie : c'est son point de vue qui est exprimé ici. La fin du discours direct rapporté est marquée par une hésitation («euh»), suivie par la justification de l'interprétation proposée : " oui peut-être parce que $i l$ ». C'est à nouveau l'énonciatrice apprenante qui s'exprime ici en expliquant son idée à ses pairs et à l'enseignante.

Dans l'exemple suivant, Andrea exprime également les paroles du personnage de la photographie :

Exemple 2

SEQ 51 :

077 Andreapeut-être l'homme il pense euh arrêtons mariage et *viva* je ne sais pas comment se dit *viva* $\uparrow$

080 Andreavivre le Pacs

$081 \quad \mathrm{P} \quad$ AH OUI vive le Pacs

082 As et $\mathrm{P}($ éclats de rires)

$083 \mathrm{P} \quad$ arrêtons le mariage vive le Pacs (rires) ok (rires)

Il s'agit de paroles intérieures : le discours direct rapporté est introduit par le verbe «il pense » (TP 77). Ici encore, « euh » joue le même rôle que les guillemets joueraient à l'écrit: il introduit les paroles rapportées «arrêtons mariage et *viva*». Cependant, les pensées du personnage sont interrompues par une focalisation sur la langue ; on observe alors le point de vue de l'apprenante qui redevient l'énonciatrice : "je ne sais pas comment se dit *viva* $\uparrow »$ (TP 77). L'utilisation de l'impératif à la première personne du pluriel ("arrêtons mariage ») est ici remarquable : le personnage, ou plutôt la moitié homme du personnage, 
s'adresse à la moitié femme avec laquelle il était censé se marier et lui propose d'annuler le mariage pour se pacser à la place : «vivre le Pacs » (TP 80).

L'attribution de paroles à un personnage situé dans le hors-champ est également possible :

Exemple 3

SEQ 51:

279 Fabiola ah oui pendant le le père de + pendant le père de le père de c'est bon $\uparrow$ pendant le père il dit d'accord euh vous êtes faits mariés jusqu'à la mort

Fabiola fait parler le prêtre qu'elle imagine dans le hors-champ : "pendant le père il dit d'accord euh vous êtes faits mariés jusqu'à la mort». La délimitation des paroles rapportées porte ici à confusion: l'adverbe «d'accord» peut être inclus ou non dans l'énoncé du prêtre. S'il n'en fait pas partie, il marque alors le changement d'énonciateur, tout comme «euh». Cependant, «d'accord» peut également être attribué au prêtre qui confirme le mariage. La locutrice Fabiola met ainsi en scène un énonciateur, le prêtre, qui prononce un acte performatif. Le discours direct rapporté permet ici à l'apprenante d'expliquer le moment auquel elle se réfère : "pendant le père ».

L’immersion fictionnelle est favorisée par la médiation des personnages. Ici, le discours direct rapporté souligne cette empathie des participants envers les sujets photographiés.

\section{2- Disparition de la frontière spectateur/personnage: les apprenants, acteurs des photographies}

Cependant, la frontière entre apprenant et personnage tend à disparaitre lorsqu'aucun verbe introductif ne précède les paroles du sujet photographié. Les étudiants incarnent alors ces personnages à la manière de comédiens. Jean-Marie Schaeffer précise que « les acteurs de théâtre et de cinéma feignent (ludiquement) une substitution d'identité actancielle » (254); ils effectuent une représentation par imitation.

La frontière entre apprenant et personnage n'est alors plus clairement définie, comme le montre l'exemple suivant :

Exemple 4

SEQ 45 :

065 Minji parce que quelqu'un ne voudrait pas se marier euh parce que \#il savait de\# les histoires de mariage c'est trop beaucoup de choses on prépare trop beaucoup de choses donc euh je voudrais plus simple euh il y a beaucoup de choses il y a beaucoup de choses qui tombées euh cassées euh 
Minji met ici en scène différents énonciateurs : elle-même observant la photographie et utilisant la troisième personne lorsqu'elle se réfère au personnage, mais également le sujet photographié s'exprimant à la première personne. Elle introduit son interprétation de la photographie : le mariage est une célébration trop compliquée. Pour cela, elle passe de l'énonciation à la troisième personne, "quelqu'un ne voudrait pas se marier ", à une énonciation focalisée sur le personnage et prononcée à la première personne : «je voudrais plus simple ». Cette irruption de la voix du sujet photographié est indiquée par deux hésitations de part et d'autre de l'énoncé : « euh je voudrais plus simple euh ».

Plus tard dans la séquence, l'apprenante se fond à nouveau dans le personnage :

Exemple 5

SEQ 45 :

219 Minji ah il voudrait euh je suis célibataire je n'aime pas beaucoup euh se marier (lève la main comme le personnage de la photo)

221 Minji euh je je pense son idée $\uparrow$

225 Minji son idée euh je voudrais (lève la main) TOUT SEUL je je n'ai pas besoin avec quelqu'un euh

$226 \quad P \quad$ ah je voudrais $\uparrow$

227 Minji tout seul

228 P me me

229 Minji célibataire (lève la main)

$230 \quad \mathrm{P} \quad$ je voudrais être célibataire $\uparrow$ je voudrais me marier tout seul $\downarrow$

231 Minji OUI je voudrais marier (lève la main) avec moi (rires)

$232 \quad \mathrm{P} \quad$ se marier tout seul $\uparrow(a ̀$ Parvine) bonjour

233 Minji oui avec euh je voudrais ma- marier avec toute seule

Elle exprime les souhaits du personnage, tantôt en se plaçant du point de vue de celuici, « je voudrais » (TP 225), tantôt en conservant son propre point de vue : « il voudrait » (TP 219), "son idée » (TP 225). La fiction émerge ici à la suite d'une hésitation : " euh je suis célibataire je suis célibataire je n'aime pas beaucoup euh se marier » (TP 219), "euh je voudrais TOUT SEUL» (TP 225), "je voudrais ma-marier avec toute seule » (TP 233). Aucun verbe de dire ne précède les paroles du personnage; cependant, celles-ci sont introduites par des expressions signifiant que l'on entre dans l'esprit du sujet photographié : « il voudrait» (TP 219), «son idée » (TP 221, 225). Minji s'approprie ici le personnage en lui attribuant des désirs et des pensées. Le mimétisme se manifeste également par la reproduction du geste du personnage. L'apprenante ne disparait toutefois pas totalement de l'énonciation : avec «je pense son idée» (TP 221), elle exprime son point de vue en tant qu'observatrice de la photographie.

Minji répète à cinq reprises dans la séquence l'idée « je voudrais tout seul » avec des 
nuances dans le sens : se marier tout seul, être célibataire ou se marier avec soi-même.

Exemple 6

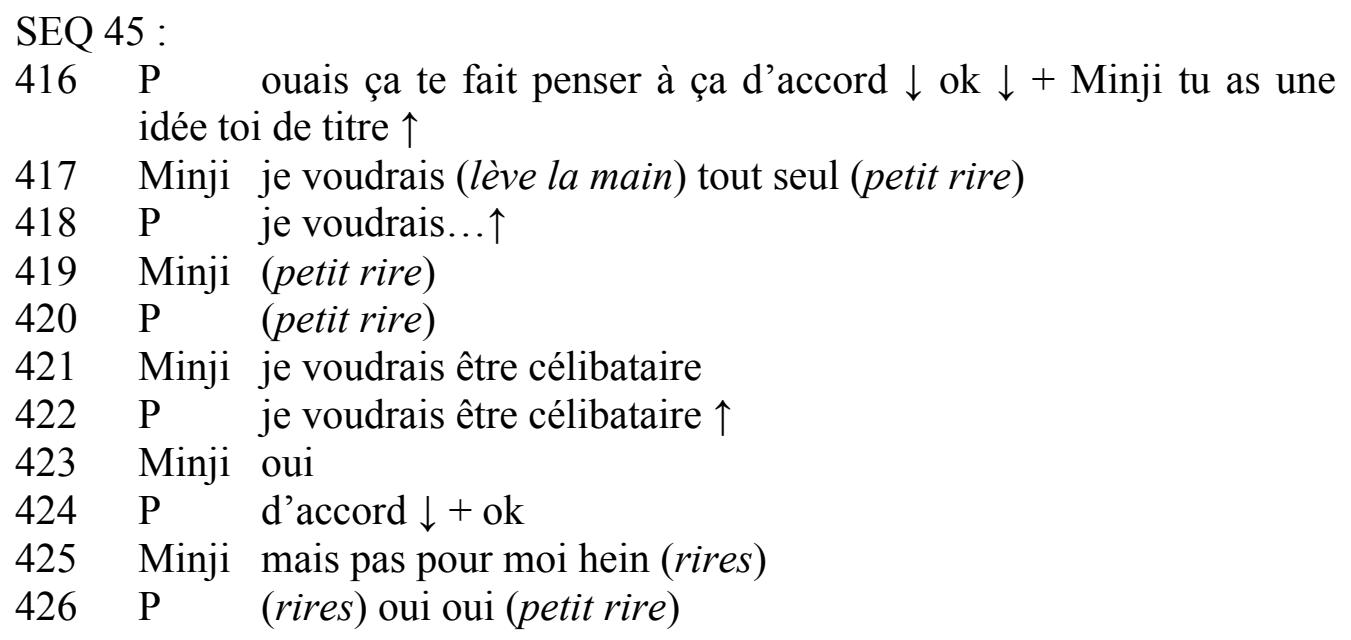

L'enseignante demande ici aux apprenants d'attribuer un titre à la photographie. Les énoncés de Minji, "je voudrais (lève la main) tout seul» (TP 417), "je voudrais être célibataire » (TP 421), constituent des actes de langage promissifs car ils sont accompagnés d'un geste de la main imitant celui du personnage en train de jurer. L'étudiante incarne le personnage en reproduisant la position de sa main et en lui prêtant sa voix. L'immersion fictionnelle est intense. Minji navigue entre le réel et l'irréel. Elle se prête au jeu : à la manière des « acteurs feignant d'être les personnages » (Searle 113), elle feint d'être le sujet photographié. Au tour de parole 425, l'apprenante prend conscience de la polyphonie ; elle se rend compte que la frontière entre monde réel et univers fictif s'efface. C'est ce qui l'amène à affirmer sa primauté en tant que locutrice : «mais pas pour moi hein ». Certes, elle met en scène un énonciateur à qui elle prête sa voix, mais elle tient à faire la distinction entre les idées qu'elle lui attribue et ses propres pensées. Minji a ici recours à un procédé «de distanciation ironique » (Schaeffer 23) pour éviter que son énoncé, qui relève de la feintise ludique, soit considéré comme une feintise sérieuse. Les rires au tour de parole 425 témoignent du changement de régime discursif : Minji retrouve son identité propre.

\section{Conclusion}

Nous avons pris pour objet d'étude les manifestations verbales d'apprenants à la vue de supports artistiques. L'immersion fictionnelle constitue une réaction émotionnelle à travers laquelle les participants s'approprient la photographie. Les étudiants s'imaginent à la place des sujets photographiés et c'est ainsi qu'ils leur attribuent des paroles. On se situe dans le domaine de la feintise ludique. Les personnages constituent un intermédiaire entre le 
monde réel et l'univers fictionnel. Mais pour que l'appropriation de la fiction par les destinataires puisse avoir lieu, les récepteurs doivent trouver une analogie entre ces deux univers. La photographie constitue en ce sens un objet de projection; c'est comme si elle tendait un miroir au spectateur, lui permettant de voir son reflet dans les images. L'entrée dans l'univers fictionnel, qui apparait à l'initiative des apprenants, offre aux participants la possibilité de parler de soi à travers un autre. La question de l'interculturalité apparait ici ouvertement, dans la mesure où plusieurs situations imaginées et plusieurs moi et moipersonnages coïncident lors du cours. L'immersion fictionnelle permet ainsi de concilier art et émotion en cours de langue et de favoriser une forte implication des apprenants.

\section{Bibliographie}

Beacco, Jean-Claude. L'approche par compétences dans l'enseignement des langues. Paris : Didier, 2007.

Boré, Catherine. «L'écriture scolaire : langue, norme, 'style', quelques exemples dans le discours rapporté ». LINX 51 (2004) : 91-106.

Cicurel, Francine. «L’instabilité énonciative en classe de langue : du statut didactique au statut fictionnel du discours ». Les Carnets du Cediscor 4 (1996) : 77-92.

Conseil de l'Europe. Livre blanc sur le dialogue interculturel. "Vivre ensemble dans l'égale dignité ». Strasbourg : Conseil de l'Europe, Division des politiques linguistiques, 2008.

Ducrot, Oswald. Le dire et le dit. Paris : Minuit, 1984.

Esquenazi, Jean-Pierre. La vérité de la fiction. Comment peut-on croire que les récits de fiction nous parlent sérieusement de la réalité ? Paris : Lavoisier, 2009.

Grice, Herbert Paul. « Logique et conversation ». Communications 30 (1979 [1975]) : 56-72.

Lahire, Bernard. L'homme pluriel. Les ressorts de l'action. Paris : Nathan, 2001 [1998].

Moore, Danièle et Diana-Lee Simon. «Déritualisation et identité d'apprenants ». AILE 16 (2002) : 121-143.

Muller, Catherine. «La photographie, déclencheur de parole en classe de FLE : la fiction dans les interactions ». Congrès mondial de linguistique française. Dir. J. Durand, B. Habert et B. Laks. Paris: Institut de Linguistique française, 2008. 533-546. http://www.linguistiquefrancaise.org/index.php?option=article\&access=standard\&Ite mid=129\&url=/articles/cmlf/pdf/2008/01/cmlf08049.pdf [consulté le $1^{\text {er }}$ septembre 2012]. 
---. « Rencontre interculturelle d'imaginaires : Débat sur le mariage devant la photographie Café noir et blanc de Doisneau ». Synergies Chili 5 (2009) : 59-69.

---. «Paroles sur images : Les interactions orales déclenchées par des photographies d'auteur en classe de français, langue étrangère ». Thèse de doctorat, Paris : U Sorbonne Nouvelle - Paris 3, 2011.

---. «La photographie, un outil pour communiquer en classe de langue ». Recherche et pratiques pédagogiques en langues de spécialité, Cahiers de l'APLIUT, Vol. XXXI 1 (2012) : 10-27.

Schaeffer, Jean-Marie. Pourquoi la fiction? Paris : Seuil, 1999.

Searle, John. Sens et expression. Études de théorie des actes de langage. Paris : Minuit, 1982 [1979].

Tournier, Michel et Arthur Tress. Rêves. Bruxelles : Complexe, 1979.

Trévise, Anne. « Spécificité de l'énonciation didactique dans l'apprentissage de l'anglais par des étudiants francophones ». Encrages (automne 1979) : 44-52.

Wunenburger, Jean-Jacques. L'imaginaire. Paris : PUF, 2003.

\section{Annexes}

\section{Photographie}

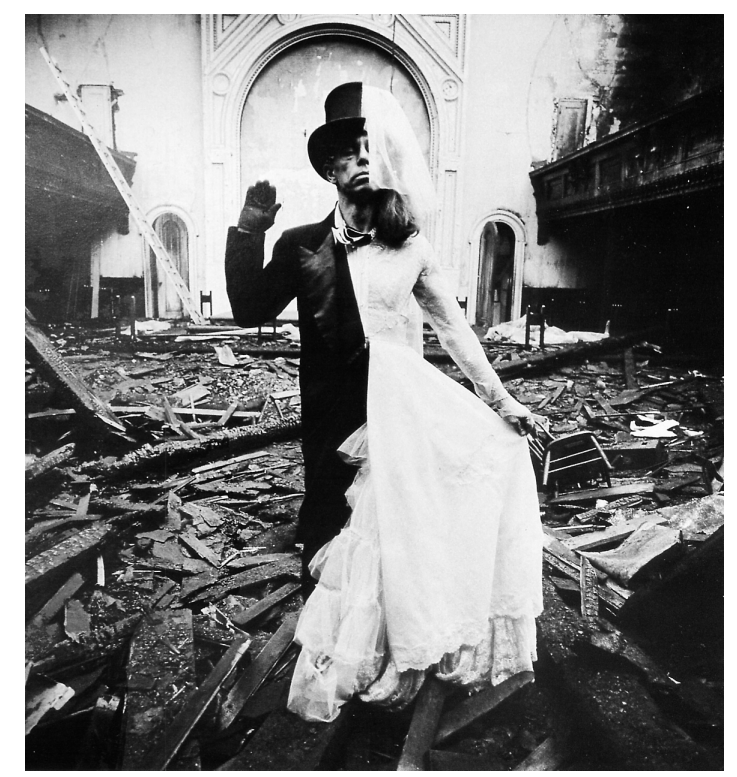

Arthur Tress, Stephen Brecht, Bride and Groom, New York, (Les mariés), 1971

(C) Arthur Tress - Reproduit avec l'aimable autorisation d'Arthur Tress 


\section{Conventions de transcription}

\begin{tabular}{|c|c|}
\hline AH OUI & emphase intonative \\
\hline bl- & mot tronqué \\
\hline maintenant & chevauchement \\
\hline *viva* & segment dans une langue autre que le français \\
\hline \#garçon\# & segment incertain \\
\hline (rires) & commentaire sur le non-verbal ou le para-verbal \\
\hline+ & pause ( 1 à 2 secondes) \\
\hline$\uparrow$ & intonation montante \\
\hline$\downarrow$ & intonation descendante \\
\hline$\ldots \uparrow$ & demande d'achèvement interactif \\
\hline $\mathrm{P}$ & professeur \\
\hline Nasiba & prénom de l'apprenant quand identifié \\
\hline As & plusieurs apprenants \\
\hline
\end{tabular}

Les prénoms des apprenants ont été modifiés en tenant compte de leur sexe et de leur nationalité.

\footnotetext{
NOTES

${ }^{1}$ La photographie, qui figure dans Michel Tournier et Arthur Tress, est présentée dans les annexes.

${ }^{2}$ L'approche qualitative adoptée dans cet article ne permet pas de déterminer la proportion des individus « réfractaires » à l'immersion fictionnelle, celle des apprenants "metteurs en scène » et celle des étudiants «acteurs» des photographies. Il serait intéressant de procéder à une étude quantitative du corpus des 51 séquences pour déterminer les grandes tendances existantes.
} 